\title{
VARIABILIDAD GENÉTICA EN POBLACIONES DE ELIONURUS MUTICUS (Poaceae) de Corrientes, Argentina, a partir de marcadores MOLECULARES DE ADN NUCLEAR Y CLOROPLÁSTICO
}

\author{
ERCILIA M. SARA MORENO ${ }^{1 *}$, NOELIA E. A. ALMIRÓN ${ }^{1}$, M. CAROLINA PEICHOTO ${ }^{3,4}$ y \\ VIVIANA G. SOLÍS NEFFA ${ }^{1,2}$
}

\begin{abstract}
Resumen: Elionurus muticus constituye un valioso recurso renovable; utilizado como forraje, fijador del suelo, aromatizante de ropa, para ahuyentar polillas, como antiséptica, contra la gonorrea y problemas urinarios, para la bronquitis, para curar la lepra, entre otros usos. Además, por su contenido en citral, se lo ha propuesto como sucedáneo de aceites esenciales, como la citronela. No obstante su potencial, se cuenta con escasos trabajos de caracterización genética de la especie. En este trabajo caracterizamos genéticamente poblaciones de E. muticus de Corrientes (Argentina), empleando marcadores moleculares RAPD y de ADNcp. Los resultados evidencian variabilidad genética en las poblaciones, y serían el resultado de la deriva genética, la historia de reducción poblacional de la especie y los modos de reproducción de la especie. Dado que en trabajos previos en poblaciones de Argentina, se observó variabilidad morfológica y en la capacidad de producir aceites, un análisis conjunto de variabilidad morfológica, genética y química en un mayor número de poblaciones, representativo de toda el área de distribución de la especie, enriquecerá el conocimiento sobre el estado de los recursos genéticos actuales de E. muticus, y contribuirá a la puesta en valor de la especie como recurso natural y como potencial cultivo de uso comercial sostenible.
\end{abstract}

Palabras clave: ADNcp, Elionurus muticus, RAPD, variabilidad genética.

Summary: Genetic variability in populations of Elionurus muticus (Poaceae) from Corrientes, Argentina, based on molecular markers of nuclear and chloroplastic DNA. Elionurus muticus constitutes a valuable renewable resource; it is used as forage, soil fixer, clothing flavoring, to drive away moths, as antiseptic, against gonorrhea and urinary problems, for bronchitis, to cure leprosy, among other uses. In addition, due to its citral content, it has been proposed as a surrogate for essential oils, such as citronella. Despite its potential, there are few works of genetic characterization of this species. In this work, we genetically characterize populations of E. muticus from Corrientes (Argentina), using molecular markers RAPD and cpDNA. Results show genetic variability in populations, which would be the results of genetic drift, a history of population reduction and the reproduction types of the species. Since, in previous works in Argentinian populations, it was found morphological variability and in the ability to produce oils, a joint analysis of morphological, chemical and genetic variability, in a bigger number of populations, representative of the entire area of distribution of the species, will enrich the knowledge about the state of the current genetic resources of E. muticus, and will contribute to the enhancement of the species as a natural resource and as a potential crop for sustainable commercial use.

Key words: ADNcp, Elionurus muticus, genetic variability, RAPD.

\footnotetext{
${ }^{1}$ Laboratorio de Citogenética y Evolución Vegetal. Instituto de Botánica del Nordeste (UNNE-CONICET). C.C. 209, 3400, Corrientes, Argentina.

${ }^{2}$ Facultad de Ciencias Exactas y Naturales y Agrimensura (UNNE).

${ }^{3}$ Laboratorio de Taxonomía. Instituto de Botánica del Nordeste (UNNE-CONICET). C.C. 209, 3400, Corrientes, Argentina.

${ }^{4}$ Facultad de Ciencias Agrarias (UNNE).

*e-mail: emsaramoreno@gmail.com
} 


\section{INTRODUCCIÓN}

Elionurus Humb. \& Bonpl. ex Willd. (Andropogoneae, Poaceae) comprende 15 especies distribuidas en áreas tropicales y subtropicales de África, Australia y América (Renvoize, 1978; Clayton \& Renvoize, 1986; Watson \& Dallwitz, 1992). El género Elionurus pertenece a la tribu Andropogoneae Dumort. y fue asignada a la subtribu Rottbolliinae J. Presl en base a su morfología (Watson \& Dallwitz, 1992) y a estudios de filogenia molecular (Soreng et al., 2015). Sin embargo, una actualización reciente de la filogenia sitúa a Elionurus en la subtribu Tripsacinae Dumort. (Soreng et al., 2017).

En América habitan 7 taxones y para la Argentina se mencionan 2 especies E. muticus (Spreng.) Kuntze y E. tripsacoides Humb. \& Bonpl. ex Willd. (Denham, 2012). Elionurus muticus es la especie del género más ampliamente distribuida. Habita desde Brasil hasta la Argentina, como así también en África. En Argentina, es posible encontrarla en las provincias de Buenos Aires, Chaco, Córdoba, Corrientes, La Pampa, Misiones, Salta, Santa Fe y Santiago del Estero. Allí se la conoce con nombres comunes tales como "pasto amargo", "pasto bravo", "espartillo", "espartillo guazú". Crece en suelos pobres y arenosos (Nicora \& Rúgolo de Agrasar, 1987); se la encuentra comúnmente en bosques abiertos del centro-oeste de Santa Fe, donde forma praderas, y en dunas y regiones arenosas de la región semiárida Pampeana, donde constituye un valioso recurso como fijadora de suelos (Anónimo, 1985). Además, es una especie dominante de las sabanas del Chaco Húmedo y de los aybales del Chaco Semiárido. En la provincia de Corrientes se la encuentra en los bosques mesófilos donde forma espartillares (Fontana, 2008).

Elionurus muticus presenta una gran variabilidad morfológica. Bajo este nombre se involucra a una serie de binomios que incluye a E. adustus (Trin.) Ekman, E. candidus (Trin.) Hack., E. latiflorus (Nees ex Steud.) Hack., E. megapotamicus (Spreng.) Herter, E. rostratus Nees y E. viridulus Hack. (Renvoize, 1978), entre otros nombres, en algunos casos sólo reconocidos por ciertos autores de trabajos regionales.

Elionurus muticus constituye un valioso recurso renovable. Con un manejo apropiado, puede emplearse como especie forrajera para alimentar el ganado (Castro \& Ramos, 2003; Hess et al., 2007). Además, se han reportado diferentes usos de esta especie en medicina popular debido a sus propiedades sudoríficas, febrífugas, afrodisíacas y antioxidantes (Dzingirai et al., 2007; Muchuweti et al., 2007). Las partes aéreas brindan un aceite esencial aromático que posee acción analgésica y antibacterial (Tredgold, 1986). Con sus hojas se realizan infusiones utilizadas para curar la lepra (Guenther, 1950). Sus espiguillas se utilizan en infusiones contra la gonorrea y problemas urinarios, así como en forma de jarabe para la bronquitis crónica de los niños (Stuckert, 1904). Presenta también actividad antifúngica, citotóxica e insecticida, por lo que se sugirió que podría emplearse en la agroindustria (Stuckert, 1904; Vidal, 1954; Cacciabue et al., 2005; Sabini et al., 2006; Dzingirai et al., 2007; Hess et al., 2007; Füller et al., 2014; Colpo et al., 2014). Otra característica interesante de E. muticus es que más del $80 \%$ de la resina de sus aceites aromáticos está compuesta por citral (Scramin et al., 2000; Füller et al., 2014). Este compuesto posee un fuerte aroma cítrico (Fester et al., 1961; Elechosa et al., 1986), motivo por el cual fue propuesto como un sucedáneo de la citronela (Fester et al., 1961; Kolb et al., 2007; Füller et al. 2014), de gran valor en las industrias aromáticas, de alimentos, cosmética y farmacéutica (Heydorn et al., 2003; Koshima et al., 2006; Hess et al., 2007; Colpo et al., 2014). Asimismo, la composición química de los aceites esenciales es muy variable en E. muticus (Chagonda et al., 2000), habiéndose observado diferencias en sus principales componentes en relación a los cambios estacionales (Hess et al., 2007) y a la procedencia geográfica de las plantas (Füller et al., 2010, 2014). La presencia del citral ha sido detectada en plantas provenientes del centro-oeste de Brasil, aunque sería más abundante en algunas poblaciones del sur de Brasil y en particular, en el nordeste argentino (Scramim et al., 2000; Cacciabue et al., 2005; Sabini et al., 2006; Kolb et al., 2007; Hess et al., 2007; Füller et al., 2010). Sin embargo, a pesar de poseer un gran potencial para su explotación comercial (Kolb et al., 2012), esta especie aún permanece subexplotada.

En este contexto, a fin de contribuir a la puesta en valor de esta especie como recurso natural y como potencial cultivo de uso comercial sostenible, hemos iniciado estudios focalizados en la caracterización 
morfológica y química de esta especie. En particular, se ha puesto un especial interés en las poblaciones del nordeste argentino (NEA), dado que en estudios preliminares se ha detectado una gran variación tanto morfológica (González et al., 2016) como en la producción de aceites aromáticos (Elechosa, 1986; Kolb et al., 2012). Además, debido que el éxito de cualquier programa de domesticación y premejoramiento depende de la variabilidad genética de las poblaciones parentales involucradas (Nodari \& Guerra, 2000), también resulta imprescindible caracterizar la variabilidad genética de las poblaciones de E. muticus. Sin embargo, la información acerca de la variabilidad genética de esta especie es aún escasa (Füller et al., 2010, 2015) y no se cuenta con datos de las poblaciones del NEA.

Sobre la base de estos antecedentes, y dado que los marcadores moleculares han contribuido con métodos efectivos para detectar diferenciación genética a nivel intra e interpoblacional en varias especies (Masi et al., 2006; Yamamoto, 2006), el objetivo del presente trabajo fue caracterizar genéticamente poblaciones naturales de Elionurus muticus de la provincia de Corrientes, Argentina, a través del uso de marcadores moleculares de ADN nuclear (ADNn) y cloroplástico (ADNcp).

\section{Materiales y Métodos}

\section{Extracción de ADN}

Un total de 24 individuos fueron muestreados en 4 poblaciones naturales de E. muticus de la provincia de Corrientes, Argentina (Fig. 1 y Tabla 1). Se colectaron hojas jóvenes y se conservaron con sílica-gel para su deshidratación y subsecuente extracción del ADN. Para cada población se obtuvieron las coordenadas geográficas utilizando una unidad de sistema de posicionamiento global (GPS). Los ejemplares de referencia fueron depositados en el herbario del Instituto de Botánica del Nordeste (CTES).

El ADN total se extrajo usando un protocolo modificado de extracción con CTAB tomado de Doyle \& Doyle (1987) y Cullings (1992).

\section{RAPD (Random Amplified Polymorphism DNA)}

Un total de 20 cebadores universales de RAPD (OPO-1 al OPO-20) de Operon Technologies
(Alameda, CA, USA) fueron evaluados. Las reacciones de amplificación se llevaron a cabo en un volumen final de $8 \mu \mathrm{l}$ con $12,5 \mathrm{ng} / \mu \mathrm{l}$ de ADN, $0,2 \mu \mathrm{M}$ de cebador, $0,10 \mathrm{mM}$ de cada dNTP, 0,19 $\mathrm{U}$ de Taq polimerasa y $0,75 \mathrm{mM}$ de $\mathrm{MgCl}_{2}$ en una solución tampón de reacción. Las amplificaciones se realizaron en un termociclador con gradiente Eppendorf Mastercycler programado con un ciclo a $94{ }^{\circ} \mathrm{C}(2 \mathrm{~min}), 35 \mathrm{ciclos}$ a $94{ }^{\circ} \mathrm{C}(30 \mathrm{seg}), 37{ }^{\circ} \mathrm{C}$ (1 min), $72^{\circ} \mathrm{C}(2 \mathrm{~min})$, y un paso final de extensión a $72{ }^{\circ} \mathrm{C}(5 \mathrm{~min})$. Los productos de amplificación se resolvieron por electroforesis en geles de agarosa al $2 \%$ a $2,3 \mathrm{~V} / \mathrm{cm}$ por $160 \mathrm{~min}$ en una solución tampón TAE $1 \times$. Luego de la electroforesis, los geles fueron teñidos con bromuro de etidio $(10 \mathrm{mg} /$ $\mathrm{ml}$ ), enjuagados por $30 \mathrm{~min}$ en agua deionizada, y fotografiados bajo luz UV. Un control negativo se incluyó en todas las reacciones.

Los productos de PCR fueron registrados como presencia (1) o ausencia (0) para construir una matriz binaria. La diversidad genética fue estimada por el número total de bandas (NTB), número de bandas exclusivas (NBE), el porcentaje de loci polimórficos (PLP), la heterocigosis esperada (He) y el índice de diversidad de Shannon (Sh) (Shannon \& Weaver, 1949). Se realizó un análisis de la varianza molecular (AMOVA; Excoffier et al., 1992) y las relaciones entre individuos fueron estudiadas con un análisis de componentes principales (PCoA) a partir de los valores de las distancias de Nei. Todos los análisis fueron llevados a cabo con el programa GenAlEx 6.3 (Peakall \& Smouse, 2006).

Identificación de poblaciones mediante análisis de agrupación Bayesiana a partir de marcadores $R A P D$

Se utilizaron métodos de agrupación Bayesiana para inferir la estructuración genética en las poblaciones estudiadas. Estos métodos permiten asignar probabilísticamente los individuos a diferentes grupos sin necesidad de conocer a priori las unidades y/o límites poblacionales (Guillot et al., 2009). Para tal fin, se aplicaron métodos basados en modelos empleando el programa STRUCTURE versión 2.2 (Pritchard et al., 2000). Se identificaron individuos agregados o mezclados ("admixed") en cada población, calculando su proporción de pertenencia a cada grupo, con un modelo de frecuencias correlacionadas (Falush et al., 2003). El 
Bol. Soc. Argent. Bot. 53 (2) 2018

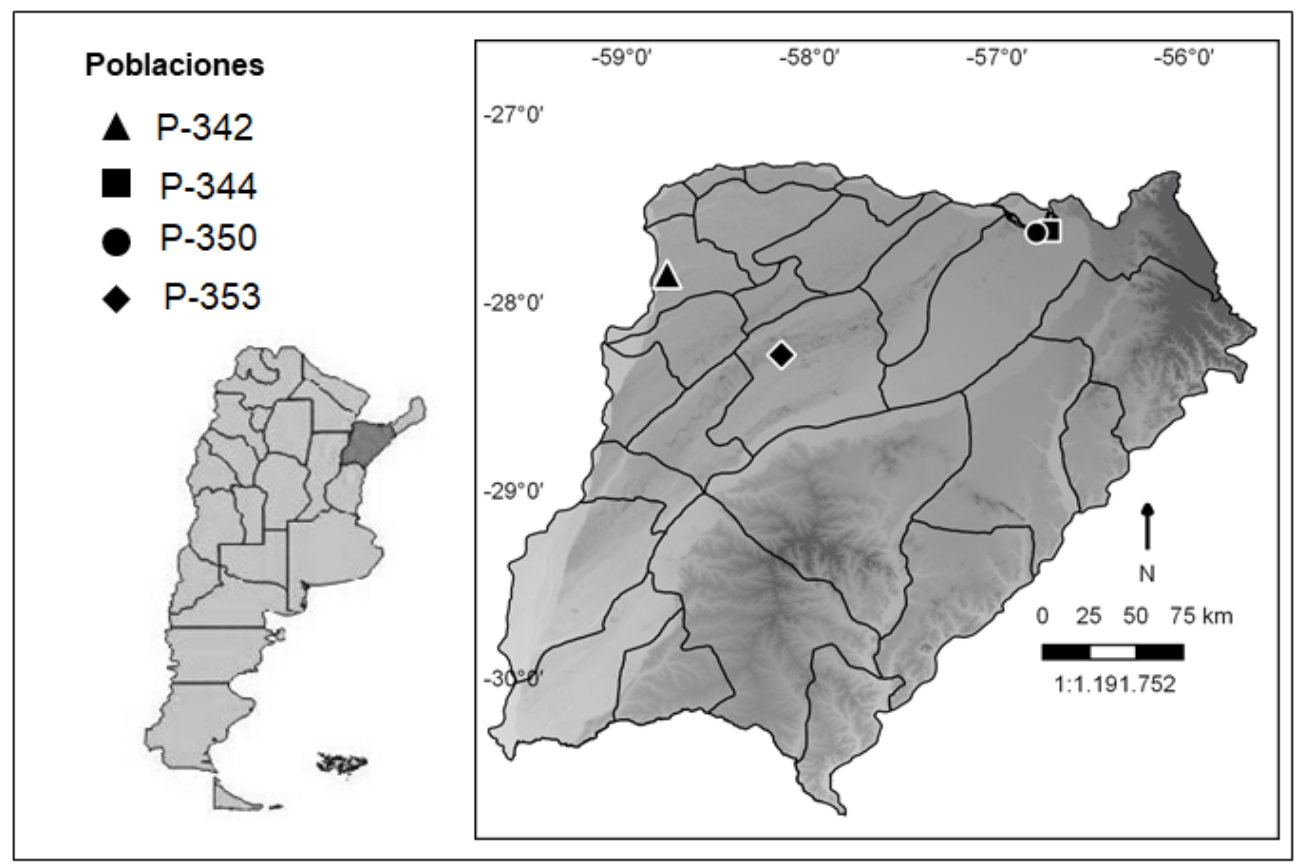

Fig. 1. Distribución de las poblaciones de Elionurus muticus de Corrientes (Argentina) analizadas.

rango de grupos posibles $(\mathrm{K})$ analizados fue de 2 a 10, llevando a cabo 3 repeticiones independientes por cada estimación de $\mathrm{K}$ para verificar la consistencia de los resultados entre los diferentes análisis. La longitud de las iteraciones de MCMC $\mathrm{y}$ el proceso de burn-in se fijaron en $1.000 .000 \mathrm{y}$ 100.000 , respectivamente. El mejor valor de $\Delta \mathrm{K}$ se estimó mediante el método de Evanno et al. (2005) mediante el programa STRUCTURE HARVESTER versión 6.94 (Dent \& Von Holdt, 2012).

\section{Tabla 1. Información de las poblaciones de Elionurus muticus analizadas y estadísticos de resumen obtenidos del análisis de marcadores moleculares RAPD y secuencias de ADNcp. NTB: número total de bandas; NBE: número de bandas exclusivas; PLP: porcentaje de loci polimórficos; He: heterocigosis esperada; Sh: índice de Shanon; P: número de sitios polimórficos; h: diversidad haplotípica; $\Pi$ : diversidad nucleotídica; DE: desvío estándar.}

\begin{tabular}{|c|c|c|c|c|c|c|c|c|c|}
\hline \multirow[b]{2}{*}{ Población } & \multirow{2}{*}{$\begin{array}{l}\text { Lugar de colección, coleccionista } \\
\text { y número de colección (herbario) }\end{array}$} & \multicolumn{5}{|c|}{ RAPD } & \multicolumn{3}{|c|}{ ADNcp } \\
\hline & & NTB & NBE & $\begin{array}{l}\text { PLP } \\
\%\end{array}$ & $\begin{array}{l}\mathrm{He} \\
(\mathrm{DE})\end{array}$ & $\begin{array}{l}\text { Sh } \\
\text { (DE) }\end{array}$ & $\mathbf{P}$ & $\begin{array}{c}h \\
(D E)\end{array}$ & $\begin{array}{c}\pi \\
(\mathrm{DE})\end{array}$ \\
\hline P-342 & $\begin{array}{l}\text { Corrientes. Dpto. Empedrado } \\
\text { (-58,76; -27,85). Peichoto M.C. } \\
\text { \& J.A. González } 342 \text { (CTES) }\end{array}$ & 58 & 4 & 49,57 & $\begin{array}{c}0,18 \\
(0,02)\end{array}$ & $\begin{array}{c}0,27 \\
(0,03)\end{array}$ & 3 & $\begin{array}{c}0,6 \\
(0,22)\end{array}$ & $\begin{array}{c}0,0012 \\
(0,0012)\end{array}$ \\
\hline P-344 & $\begin{array}{l}\text { Corrientes. Dpto. Ituzaingó (-56,71; } \\
-27,62) \text {. Peichoto M.C. } 344 \text { (CTES) }\end{array}$ & 59 & 5 & 50,43 & $\begin{array}{c}0,14 \\
(0,02)\end{array}$ & $\begin{array}{c}0,22 \\
(0,02)\end{array}$ & 3 & $\begin{array}{c}0,73 \\
(0,16)\end{array}$ & $\begin{array}{c}0,0016 \\
(0,0015)\end{array}$ \\
\hline P-350 & $\begin{array}{l}\text { Corrientes. Dpto. Ituzaingó (-56,79; } \\
-27,63) \text {. Peichoto M.C. } 350 \text { (CTES) }\end{array}$ & 70 & 7 & 58,26 & $\begin{array}{c}0,18 \\
(0,02)\end{array}$ & $\begin{array}{c}0,28 \\
(0,03)\end{array}$ & 1 & 0 & 0 \\
\hline P-353 & $\begin{array}{l}\text { Corrientes. Dpto. Concepción }(-58,15 \text {; } \\
-28,28) \text {. Peichoto M.C. } 353 \text { (CTES) }\end{array}$ & 50 & 11 & 40,87 & $\begin{array}{c}0,15 \\
(0,02)\end{array}$ & $\begin{array}{c}0,22 \\
(0,03)\end{array}$ & 1 & 0 & 0 \\
\hline
\end{tabular}




\section{E. M. S. Moreno et al. - Variabilidad genética en Elionurus muticus}

\section{Secuencias de ADN cloroplástico}

La región correspondiente al espaciador intergénico del ADN cloroplástico matK5`RmatK6 fue amplificada por medio de reacciones de PCR y empleando los cebadores de Shaw et al. (2005). Las PCR fueron llevadas a cabo en un volumen final de $25 \mu \mathrm{l}$ conteniendo $9 \mathrm{ng} / \mu \mathrm{l}$ de ADN $, 0,1 \mu \mathrm{M}$ de cada cebador, $200 \mu \mathrm{M}$ de cada dNTP, $3 \mathrm{mM} \mathrm{MgCl}_{2}$ y $2 \mathrm{U}$ de Taq polimerasa en una solución tampón de reacción $1 \times$. El programa de amplificación consistió en un ciclo inicial de 5 min a $95{ }^{\circ} \mathrm{C}$, seguido por 31 ciclos de 1 $\min$ a $95{ }^{\circ} \mathrm{C}, 1 \min$ a $50,2{ }^{\circ} \mathrm{C}$ y $1 \min$ a $72{ }^{\circ} \mathrm{C}$, seguido por un paso de extensión final de $4 \mathrm{~min}$ a $72{ }^{\circ} \mathrm{C}$. Las amplificaciones fueron confirmadas por electroforesis en geles de agarosa $1,4 \%$ en solución tampón TAE $1 \times$, teñidos en bromuro de etidio $(10 \mathrm{mg} / \mathrm{ml})$ y fotografiados bajo luz UV.

Los productos de los cebadores directo y reverso fueron enviados a secuenciar a Macrogen (C) (Seúl, Corea del Sur). Todas las secuencias fueron depositadas en el GenBank (Números de accesión: MH285961 al MH285974). Los cromatogramas fueron editados manualmente con Chromas 2.33 (http://www.technelysium. com.au). Las secuencias fueron alineadas de manera automática con MEGA 5 (Tamura et al., 2011) usando el método Clustal W. Todas las posiciones polimórficas detectadas en el alineamiento fueron chequeadas con los cromatogramas originales, con el fin de asegurar que dichas bases realmente constituyen posiciones variables. Debido a que la dinámica mutacional de las inserciones/deleciones (indels) involucrando poli-T/A no puede ser determinada (Aldrich et al., 1988; Kelchner, 2000), estos eventos no fueron considerados para los análisis posteriores. Aquellos indels contiguos fueron tratados como un único evento mutacional (Simmons \& Ochoterena, 2000).

A partir de las secuencias de ADNcp se calcularon los estadísticos descriptivos estándar para diversidad molecular: número de sitios polimórficos (P), diversidad haplotípica (h) y diversidad nucleotídica $(\pi)$. La estructura genética poblacional fue establecida con un AMOVA. Se utilizó el programa Arlequin v. 3.5.1.2 (Excoffier $\&$ Lischer, 2010). Se determinaron los haplotipos con el programa DnaSP v.5.0 (Librado \& Rozas, 2009) y las relaciones evolutivas entre los mismos se establecieron mediante la construcción de una red de haplotipos con el método median-joining ( $\varepsilon=0$, Bandelt et al., 1999), empleando el programa NETWORK 4.5.1 (http://www.fluxusengineering.com).

\section{Resultados}

\section{$R A P D$}

Cuatro cebadores fueron seleccionados para el análisis (OPO-1, -3, -10, -12) por mostrar mayor polimorfismo y calidad de las bandas, registrándose un total de 115 bandas. Los índices de diversidad genética calculados se muestran en la Tabla 1 . Se detectaron entre 50 (P-353) y 70 (P-350) bandas a nivel poblacional. Todas las poblaciones mostraron bandas exclusivas, desde 4 (P-342) hasta 11 bandas (P-353). El PLP total fue de 49,78\%, encontrándose valores desde 40,87\% (P-353) hasta 58,26\% (P-350); la He total fue de 0,16, con valores de 0,18 (P-342 y P-350) hasta 0,14 (P-344); el índice de Shannon total fue de 0,25 , con un rango de variación desde 0,22 (P-353) hasta 0,28 (P-350).

Del total de la variabilidad genética encontrada, $79 \%$ pertenece a cada población, mientras que 21 $\%$ corresponde a la variabilidad interpoblacional. En cuanto al AMOVA, el índice de fijación $\Phi_{\mathrm{ST}}$ fue significativo e igual a 0,21 (Tabla 2). Todos los valores de distancia genética entre poblaciones fueron estadísticamente significativos (Tabla 3), y variaron entre 0,088 y 0,361 . La menor distancia genética se observó entre las poblaciones P-342 y $\mathrm{P}-350$. Mientras que las poblaciones genéticamente más distantes fueron P-342 y P-353.

Las primeras dos coordenadas del PCoA representaron el $30,53 \%$ de la variabilidad $(17,60$ y $12,93 \%$ respectivamente). Los individuos de la población P-353 fueron los únicos que se agruparon claramente, aunque muy relacionados con algunos individuos de la población P-344. Los individuos de las demás poblaciones formaron dos grupos separados, uno conteniendo individuos de las poblaciones P-342 y P-350, y otro con individuos de todas las poblaciones excepto P-353 (Fig. 2).

La agrupación Bayesiana arrojada por STRUCTURE fue concordante con los resultados del PCoA en cuanto a la agrupación de los individuos de la población P-353 y su cercanía con algunos individuos de la población P-344. No 


\begin{tabular}{|c|c|c|c|c|c|}
\hline Marcador & $\begin{array}{l}\text { Fuente de } \\
\text { variación }\end{array}$ & g.l. & $\begin{array}{l}\text { Suma de } \\
\text { cuadrados }\end{array}$ & $\begin{array}{l}\text { Componentes } \\
\text { de la varianza }\end{array}$ & $\begin{array}{l}\text { Porcentaje } \\
\text { de variación }\end{array}$ \\
\hline \multirow{4}{*}{ RAPD } & $\begin{array}{c}\text { Entre } \\
\text { poblaciones }\end{array}$ & 3 & 109,08 & 3,74 & 21 \\
\hline & $\begin{array}{c}\text { Dentro de } \\
\text { poblaciones }\end{array}$ & 20 & 278,17 & 13,91 & 79 \\
\hline & Total & 23 & 387,25 & 17,65 & \\
\hline & \multicolumn{5}{|c|}{ Índice de fijación $\Phi S T=0,21^{*}$} \\
\hline \multirow{4}{*}{ ADNcp } & $\begin{array}{c}\text { Entre } \\
\text { poblaciones }\end{array}$ & 3 & 3,71 & 0,17 & 47,59 \\
\hline & $\begin{array}{c}\text { Dentro de } \\
\text { poblaciones }\end{array}$ & 20 & 3,83 & 0,19 & 52,41 \\
\hline & Total & 23 & 7,54 & 0,37 & \\
\hline & \multicolumn{5}{|c|}{ Índice de fijación $\Phi S T=0,48^{*}$} \\
\hline
\end{tabular}

obstante separó a las poblaciones analizadas en dos grupos ( $\mathrm{K}=2$; Fig. 3), a diferencia del PCoA donde se observaron tres grupos. Un grupo compuesto por los individuos de la población P-350 y la mayoría de los individuos de la población P-344, y otro grupo conformado por las poblaciones P-342, P-350, y los dos individuos restantes de la población P-344.

\section{Secuencias de ADN cloroplástico}

Las secuencias alineadas presentaron 555 posiciones. Se observaron 3 sitios polimórficos (posiciones nucleotídicas: \#250 - \#392 - \#468); se obtuvieron valores de diversidad haplotípica relativamente altos $(h=0,59)$, y valores bajos de diversidad nucleotídica $(\pi=0,0012)$ (Tabla 1$)$. Dos poblaciones no mostraron variabilidad genética (P-350 y P-353), y las dos restantes (P-342 y $\mathrm{P}-344)$ mostraron valores similares de diversidad (Tabla 1). El AMOVA fue estadísticamente significativo y reveló que el $52,42 \%$ de la variabilidad es explicada por las diferencias dentro de las poblaciones (Tabla 2). La reconstrucción de los haplotipos para el total de individuos analizados resultó en 4 haplotipos diferentes (Fig. 4). Sólo dos poblaciones presentaron haplotipos exclusivos, es decir, presentes sólo en esa población. El haplotipo H1 fue exclusivo de la población $\mathrm{P}-342$, y el haplotipo $\mathrm{H} 4$ fue exclusivo de la población P-344. Las poblaciones P-350 y P-353 presentaron un solo haplotipo cada una, $\mathrm{H} 3$ y $\mathrm{H} 2$ respectivamente. La red de haplotipos presentó forma de estrella, con un haplotipo central del cual derivan los demás haplotipos, y con un único paso mutacional separando a cada uno de los haplotipos.

Tabla 3. Matriz de distancias genéticas entre las poblaciones de Elionurus muticus analizadas en base a los datos del marcador molecular RAPD. Por encima de la diagonal se muestran los valores $P$ de significancia en base a 999 permutaciones.

\begin{tabular}{|ccccc|}
\hline P-342 & P-344 & P-350 & P-353 & Poblaciones \\
0 & 0,006 & 0,034 & 0,001 & P-342 \\
0,188 & 0 & 0,009 & 0,003 & P-344 \\
0,088 & 0,129 & 0 & 0,003 & P-350 \\
0,361 & 0,198 & 0,283 & 0 & P-353 \\
\hline
\end{tabular}




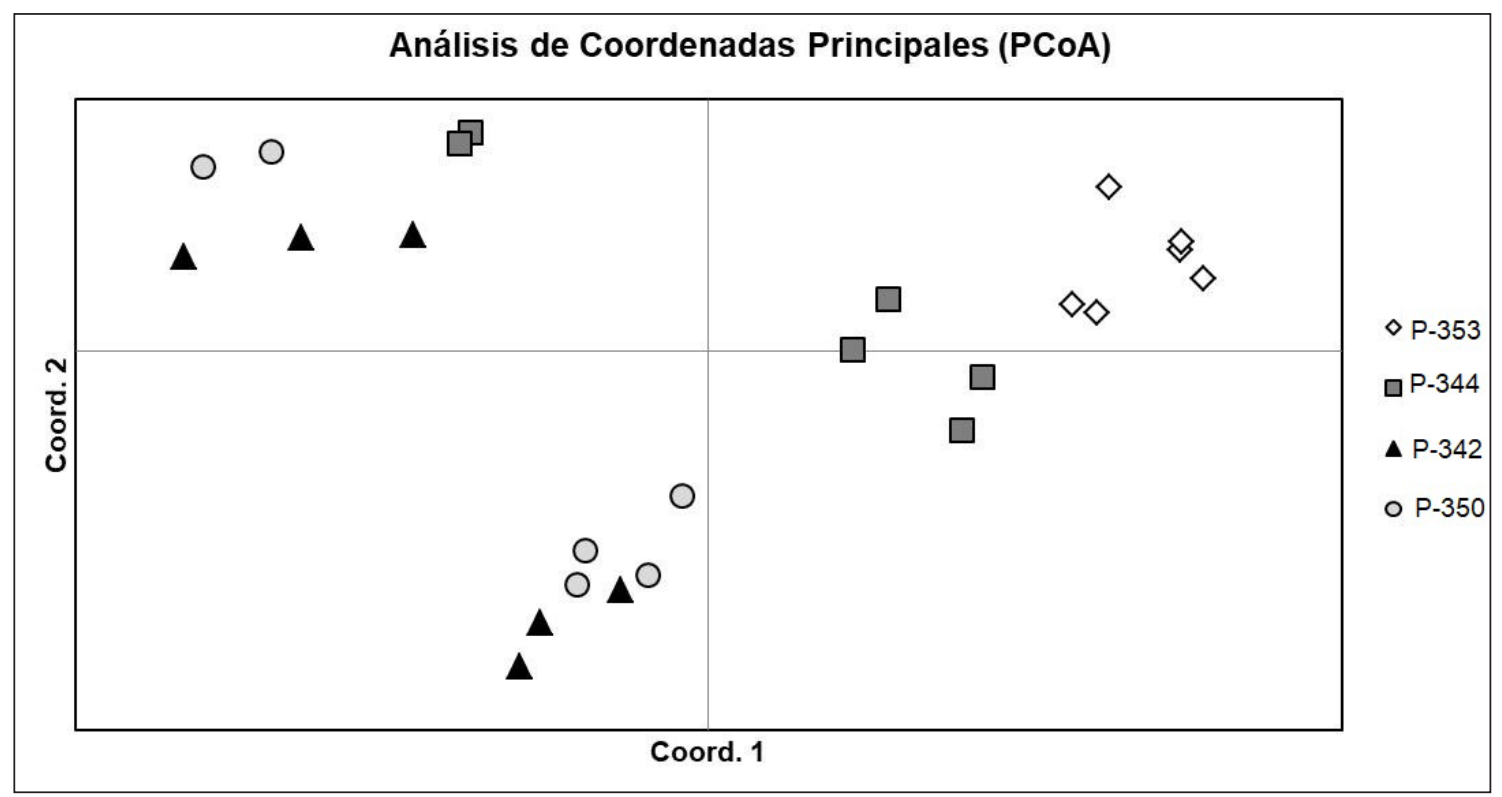

Fig. 2. Gráfico resultante del Análisis de Coordenadas Principales (PCoA) obtenido a partir de los patrones de marcadores RAPD de los individuos de Elionurus muticus analizados. Cada población se encuentra diferenciada por un símbolo.

\section{Discusión}

La caracterización de la variabilidad genética de las poblaciones de especies con potencial valor económico constituye una información básica para el desarrollo de programas de domesticación y premejoramiento (Nodari \& Guerra, 2000). Si bien existe un creciente interés en develar la variabilidad y estructura genética de plantas bioactivas (Shasany et al., 2005; Muchugi et al., 2008; Domyati et al., 2011; Azizi et al., 2012), esta información muchas veces resulta escasa. En particular, en E. muticus, la única información disponible respecto a su variabilidad genética corresponde a investigaciones llevadas a cabo en poblaciones de Rio Grande do Sul, Brasil (Füller et al., 2010, 2015). Por lo tanto, nuestro análisis de poblaciones naturales de E. muticus de la provincia de Corrientes empleando marcadores moleculares nucleares (RAPD) y secuencias de regiones no codificantes de ADNcp, constituye el primer aporte al conocimiento de la variabilidad y estructura genética de poblaciones de Argentina y aporta información para futuros estudios aplicados en esta especie.
Todos los valores de diversidad genética obtenidos en el presente trabajo a partir de marcadores RAPD resultan más bajos respecto de los obtenidos por Füller et al. (2010) empleando los mismos marcadores en poblaciones de Brasil. En cuanto a la distribución de la variabilidad genética, el análisis de coordenadas principales sólo arrojó un grupo relativamente homogéneo que incluyó todos los individuos de la población de Concepción (P-353), que es la población que presentó el mayor número de bandas exclusivas $(\mathrm{NBE}=11$ vs. $\mathrm{NBE}=4-7)$, junto con la mayoría de individuos de una población de Ituzaingó (P-344). Los restantes individuos conformaron otros dos grupos heterogéneos.

En cuanto al análisis de secuencias de ADNcp, los valores relativamente elevados de diversidad haplotípica y bajos de diversidad nucleotídica detectados en las poblaciones polimórficas de $E$. muticus, son indicativos de un rápido crecimiento poblacional, un tamaño efectivo $(\mathrm{Ne})$ bajo o de poblaciones con poco tiempo de separación (Schaal et al., 1998). 


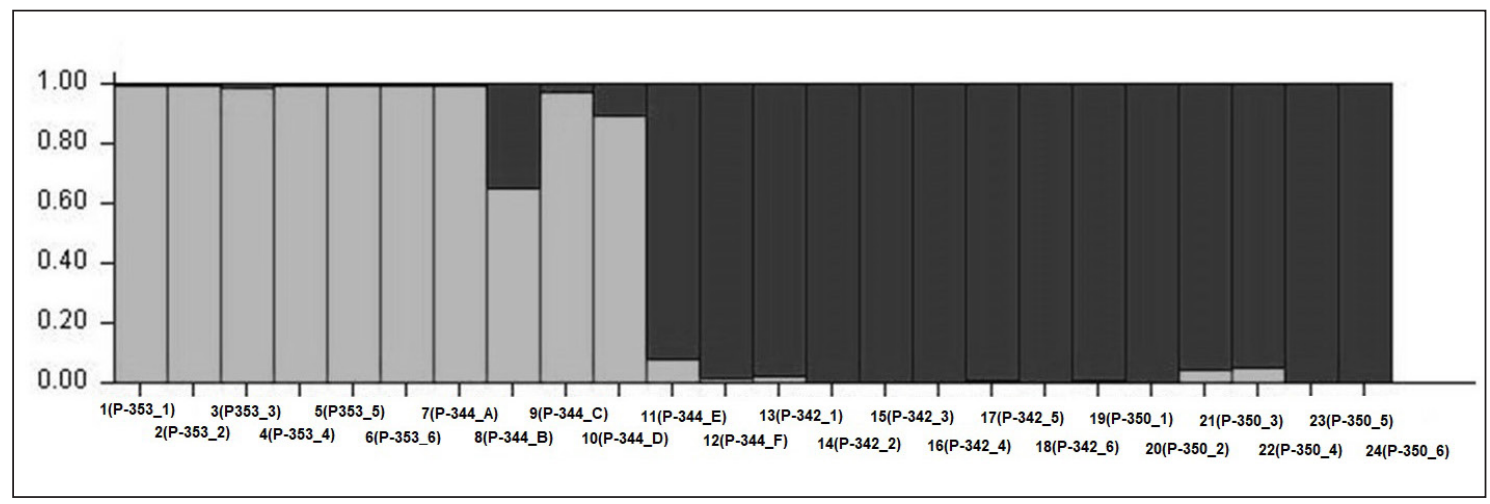

Fig. 3. Representación de las agrupaciones Bayesianas para $K=2$. Cada tono de gris indica un grupo genético. Las poblaciones se indican entre paréntesis.

Con respecto a la estructuración de la variabilidad genética, el mayor porcentaje de variabilidad intrapoblacional que interpoblacional detectada para ambos marcadores en las poblaciones de

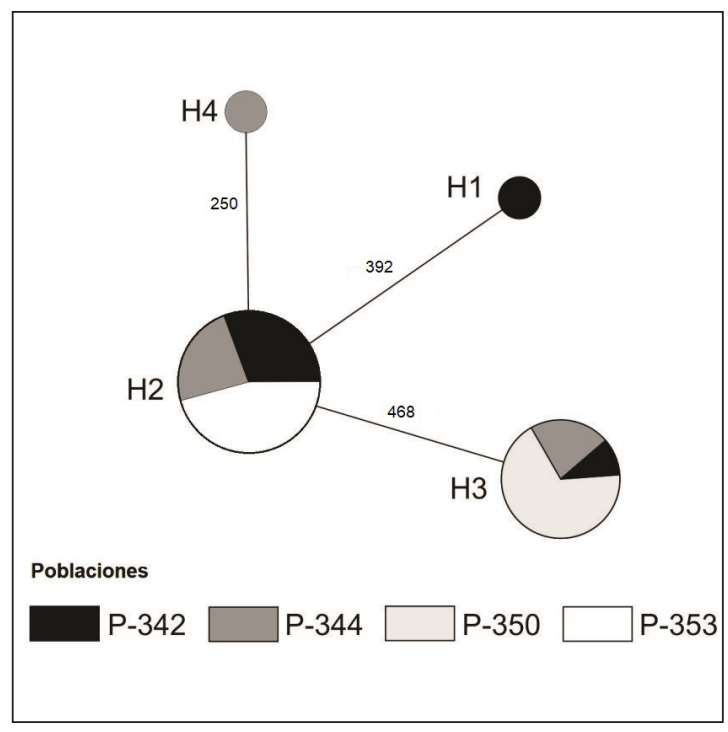

Fig. 4. Red de haplotipos obtenida a partir del análisis de secuencias de ADNcp no codificantes, mostrando las relaciones evolutivas entre los haplotipos hallados en las poblaciones de Elionurus muticus analizadas. Cada círculo representa un haplotipo, su tamaño es proporcional a su frecuencia. Las líneas que unen a los haplotipos representan sus relaciones evolutivas. Los números sobre las líneas indican la posición nucleotídica en la que se encuentra la mutación.
Corrientes de E. muticus es similar al observado en las poblaciones de Rio Grande do Sul (Füller et al., 2010, 2015). Los resultados obtenidos en las poblaciones estudiadas de Corrientes sustentan la ocurrencia de alogamia en E. muticus como también fuera propuesto para las poblaciones brasileras en base a los índices de diversidad obtenidos a partir de marcadores AFLP (Füller et al., 2015). No obstante, los valores de $\Phi$ st para ambos marcadores moleculares aquí estudiados también evidenciaron un alto grado de diferenciación genética entre las poblaciones de Corrientes. Asimismo, y contrariamente a lo observado en las poblaciones de Brasil, en las cuales era de esperarse un valor de Фst relativamente elevado debido a las distancias geográficas entre las poblaciones analizadas (Füller et al., 2015), en el presente trabajo los valores de distancia genética no reflejaron las distancias geográficas entre las poblaciones, ya que las dos poblaciones geográficamente más cercanas mostraron valores más altos de distancia genética que aquellas geográficamente más distantes. Por lo tanto, la diferenciación genética observada actualmente entre las poblaciones estudiadas de $E$. muticus en Corrientes no puede ser explicada por un modelo de flujo génico de aislamiento por distancia. Dado que las regiones de ADN no codificante, como las analizadas en este trabajo, pueden acumular cambios mutacionales de manera neutral de modo tal que únicamente la deriva génica influye en los niveles de polimorfismo en una población (Parker et al., 1998), la diferenciación observada entre las poblaciones de Corrientes podría ser el resultado 
de la deriva génica. Además, se ha sugerido que el modo de reproducción afecta la estructura genética de las plantas, dentro y entre poblaciones (Loveless \& Hamrick, 1984). Si bien E. muticus se propaga por semillas, también puede multiplicarse vegetativamente por división de rizomas (Davies et al., 2004; da Silva Nunes, 2008), de modo que las poblaciones estarían integradas por una cierta proporción de plantas clonales, hecho que también pudo contribuir al grado de estructuración genética observado.

A su vez, la estructuración genética detectada en las poblaciones de E. muticus estudiadas a partir del análisis de las secuencias de ADNcp fue mayor que la detectada a partir de marcadores nucleares. En la mayoría de las Angiospermas el ADNcp es heredado por vía materna, este hecho puede limitar el flujo génico interpoblacional a través de las semillas, contribuyendo así a mantener la estructura genética establecida durante la supervivencia en refugios y/o la colonización de nuevos hábitats (Petit et al. 1993; Comes \& Kadereit, 1998). Por lo tanto, además de poseer una baja tasa mutacional, el ADNcp puede reflejar procesos históricos, como migraciones pasadas o dinámicas de colonización durante largos períodos de tiempo (ej. Magri et al., 2006; Eidesen et al., 2007). Sumado a esto, el menor tamaño efectivo $(\mathrm{Ne})$ del genoma plastidial comparado con el del genoma nuclear, puede contribuir a incrementar la acción de la deriva génica (Ennos, 1994; Mc Cauley, 1995; El Mousadik \& Petit, 1996). Dichas características explicarían las diferencias en la estructuración genética detectada en las poblaciones estudiadas de E. muticus a partir del análisis de las secuencias de ADNcp y de marcadores nucleares.

Por otra parte, el análisis de la red de haplotipos de ADNcp evidencia un origen genético común de las poblaciones de E. muticus, con un haplotipo central (H2), el que podría señalarse como el haplotipo ancestral a partir del cual surgieron los demás haplotipos. Además, los resultados aquí obtenidos sugieren un origen muy reciente de los haplotipos, ya que un único paso mutacional separa a cada uno de ellos, siendo la mayoría haplotipos de punta. Estos resultados son consistentes con los índices de diversidad haplotípica y nucleotídica obtenidos y reflejan una historia evolutiva reciente para las poblaciones analizadas, o bien un proceso de rápida expansión poblacional, luego de que poblaciones con un $\mathrm{Ne}$ pequeño atravesaran un evento tipo cuello de botella.

En síntesis, los resultados aquí obtenidos evidencian la presencia de variabilidad genética en las poblaciones naturales estudiadas de E. muticus de la provincia de Corrientes. Los valores de variabilidad genética observados podrían ser el reflejo tanto de una historia de reducción poblacional de la especie, como de sus modos de reproducción.

Asimismo, dado que en estudios preliminares se distinguieron grupos de poblaciones en la provincia de Corrientes que diferían en la longitud de las espiguillas y en el diámetro de las cañas (González et al., 2016) y que, aparentemente, dichas poblaciones también diferirían en su capacidad de producir aceites esenciales (Peichoto et al., inéd.), un análisis conjunto de la variación morfológica, genética y química, de un número mayor de poblaciones representativa de toda el área de distribución geográfica de la especie, podría contribuir al conocimiento sobre el estado de los recursos genéticos presentes en las poblaciones naturales de E. muticus, y posteriormente aplicar dicho conocimiento a planes de pre-mejoramiento.

\section{Agradecimientos}

Los autores agradecen las sugerencias realizadas por los revisores que contribuyeron a mejorar el manuscrito. Este trabajo fue financiado por P004/14 (SGCyT-UNNE), PI A013-2013 (SGCyT-UNNE) y PICT $2012 \mathrm{~N}^{\circ} 1812$ (ANPCyT-FONCyT). M. C. Peichoto y V. G. Solís Neffa son miembros de la Carrera del Investigador Científico del CONICET.

\section{Bibliografía}

ALDRICH, J., B. W. CHERNEY, E. MERLIN \& L. CHRISTOPHERSON. 1988. The role of insertion/ deletions in the evolution of the intergenic region between $p s b \mathrm{~A}$ and $t r n \mathrm{H}$ in the chloroplast genome. Curr. Genet. 14: 137-46.

ANÓNIMO. 1985. Uso del "pasto amargo" forrajero. Diario Clarín, 3-VIII.

AZIZI, A., J. HADIAN, M. GHOLAMI, W. FRIEDT \& B. HONERMEIER. 2012. Correlations between genetic, morphological, and chemical diversities in a germplasm collection of the medicinal plant Origanum vulgare L. Chem. Biodivers. 9: 27842801. 
BANDELT, H. J., P. FORSTER \& A. RÖHL. 1999. Median-joining networks for inferring intraspecific phylogenies. Mol. Biol. Evol. 16: 37-48.

CACCIABUE, M., N. GALLUCCI, G. P. CORDERO, N. KOLB, M. DEMO \& L. SABINI. 2005. Elionurus muticus from north of Argentina: evaluation of the antibacterial activity of three essential oil chemotypes. Biocell 29: 223-226.

CASTRO, L. O. \& R. L. D. RAMOS. 2003. Principais gramíneas produtoras de óleos essenciais: Cymbopogon citratus, capim-cidró, Cymbopogon martinii (Rox.) J.F. Watson, palmarosa, Cymbopogon nardus (L.) Rendle, citronela, Elyonurus candidus (Trin.) Hack., capim-limão, Vetiveria zizanioides Nash, vetiver. Fepagro 11: $1-31$.

CHAGONDA, L. S., C. MAKANDA \& J. C. CHALCHAT. 2000. The essential oils of wild and cultivated Cymbopogon validus (Stapf) Stapf ex Burtt Davy and Elionurus muticus (Spreng.) Kunth from Zimbabwe. Flavour Frag. J. 15: 100-104.

CLAYTON, W. D. \& S. A. RENVOIZE. 1986. Genera Graminun. Grasses of the World. Kew Bull., Additional Series XIII. Her Majesty's Stationery Office, London.

COLPO J. F., S. M. JAHNKE \& T. FÜLLER. 2014. Potencial inseticida de óleos de origem vegetal sobre Grapholita molesta (Busck) (Lepidoptera: Tortricidae) Revista Bras. Pl. Med. 16: 182-188.

COMES, H. P. \& J. W. KADEREIT. 1998. The effect of Quaternary climatic changes on plant distribution and evolution. Trends Plant Sci. 3: 432-438.

CULLINGS, K. W. 1992. Design and testing of a plant-specific PCR primer for ecological and evolutionary studies. Mol. Ecol. 1: 233-240.

DA SILVA NUNES, A. C. G. 2008. Coleta, prospecçao em herbários e estudos sobre propagaçao vegetativa de capim limão (Elionurus sp.). Dissertação apresentada como um dos requisites à obtenção do Grau de Mestre em Fitotecnia Área de Concentração Horticultura. Porto Alegre (RS).

DAVIES, P., J. J. VILLAMIL \& R. ASHFIELD. 2004. Fichas técnicas de cultivo. En: Estudios en domesticación y cultivo de especies medicinales y aromáticas nativas. Serie FPTA-INIA. Canelones, Uruguay. 6: 35-115.

DENHAM, S. S. 2012. Elionurus. En: ZULOAGA, F. O., Z. E. RÚGOLO \& A. M. ANTON (Eds.). Flora Argentina. Plantas Vasculares de la República Argentina. Monocotiledoneae: Poaceae: Aristidoideae-Pharoideae. 3: 514-516.

DENT, A. E. \& B. M. VON HOLDT. 2012. Structure Harvester: a website and program for visualizing STRUCTURE output and implementing the Evanno method. Conserv. Genet. Resour. 4: 359-361.
DOMYATI, F. M., R. A. A. YOUNIS, S. EDRIS, A. MANSOUR, A. SABIR \& A. BAHIELDIN. 2011. Molecular markers associated with genetic diversity of some medicinal plants in Sinai. J. Med. Plant Res. 5: 200-210.

DOYLE, J. J. \& J. L. DOYLE. 1987. A rapid DNA isolation procedure for small quantities of fresh leaf tissue. Phytochem. Bull. 19: 11-15.

DZINGIRAI, B., M. MUCHUWETI, T. MURENJE, C. CHIDEWE, M. A. N. BENHURA \& L. S. CHAGONDA. 2007. Phenolic content and phospholipids peroxidation inhibition by methanolic extracts of two medicinal plants: Elionurus muticus and Hypoxis hemerocallidea. Afr. J. Biochem. Res. 1: $137-41$.

EIDESEN, P. B., I. G. ALSOS, M. POPP, Ø. STRENSRUD, J. SUDA \& C. BROCHMANN. 2007. Nuclear versus plastid data: complex Pleistocene history of a circumpolar key species. Mol. Ecol. 16: 3902-3925.

ELECHOSA M., I. MIZRAHI, M. JUAREZ \& A. BANDONI. 1986. Composición del aceite esencial de un espartillo (Elionurus muticus (Sprengel) O. Kuntze) tipo geraniol proveniente de Corrientes. S.A.I.P.A. 7: 25-39.

EL MOUSADIK, A. \& R. J. PETIT. 1996. Chloroplast DNA phylogeography of the argan tree of Morocco. Mol. Ecol. 5: 547-555.

ENNOS R. A. 1994. Estimating the relative rates of pollen and seed migration among plant populations. Heredity 72: 250-259.

EVANNO, G., S. REGNAUT \& J. GOUDET. 2005. Detecting the number of clusters of individuals using the software structure: a simulation study. Mol. Ecol. 14: 2611-2620.

EXCOFFIER, L., P. E. SMOUSE \& J. M. QUATTRO. 1992. Analysis of molecular variance inferred from metric distances among DNA haplotypes: application to human mitochondrial DNA restriction data. Genetics. 131: 479-494.

EXCOFFIER, L. \& H. E. L. LISCHER. 2010. Arlequin suite ver 3.5: a new series of programs to perform population genetics analyses under Linux and Windows. Mol. Ecol. Res. 10: 564-567.

FALUSH, D., M. STEPHENS \& J. K. PRITCHARD. 2003. Inference of population structure using multilocus genotype data: linked loci and correlated allele frequencies. Genetics 164: 1567-1587.

FESTER, G. A., E. A. MARTINUZZI, J. A. RETAMAR \& A. RICCIARDI. 1961. Aceites esenciales de la República Argentina. Academia nacional de Ciencias, Cordoba.

FONTANA, J. L. 2008. Vegetación y diversidad de ambientes en la Reserva Natural Isla Apipé Grande, Provincia de Corrientes, Argentina. INSUGEO, Miscelánea, 17: 407-424. 


\section{E. M. S. Moreno et al. - Variabilidad genética en Elionurus muticus}

FÜLLER, T. N., C. TESSELE, I. B. I. DE BARROS \& J. F. BARBOSA-NETO. 2010. Phenotypical, phytochemical and molecular characterization of capim carona [Elionurus muticus (Spreng.) Kuntze] populations. Revista Bras. Plantas Med. 12: 261-268.

FÜlLER, T. N., C. BERTRAND, A. SIMON, I. B. I. DE BARROS \& J. F. BARBOSA-NETO. 2014. Elionurus muticus as an alternative source of citral from Pampa biome, Brazil. J. Oleo Sci. 63: 11091116.

FÜLLER, T. N., D. GUERRA, M. T. SCHIFINOWITTMANN, C. TESSELE, I. BERGMAN INCHAUSTI DE BARROS \& J. FERNANDES BARBOSA NETO. 2015. Genetic and cytogenetic structure of wild lemon grass (Elionurus muticus) populations. Crop Breed Appl. Biotechnol. 15: 272277.

GONZALEZ, J. A., N. E. A. ALMIRÓN, E. M. S. MORENO, S. I. VASQUEZ, V. G. SOLÍS NEFFA \& M. C. PEICHOTO. 2016. Variabilidad morfológica y genética en Elionurus muticus (Poaceae, Panicoideae, Andropogoneae). Reunión de Comunicaciones Científicas, Técnicas y de Extensión, Facultad de Ciencias Agrarias-UNNE, Corrientes.

GUENTHER, E. 1950. The essencial oil. IV D. Van Nostrand Company, New York.

GUIllot, G., R. LEBloIS, A. COUlON \& A. C. FRANTZ. 2009. Statistical methods in spatial genetics. Mol. Ecol. 18: 4734-4756.

HESS, S. C, M. T. L. P. PERES, A. L. BATISTA, J. P. RODRIGUES, S. C. TIVIROLI, L. G. L. OLIVEIRA, C. W. C. SANTOS, L. E. S. FEDEL, S. A. M. CRISPIM, A. SMANIA JUNIOR, E. F. A. SMANIA, A. FLACH \& S. PANTAROTO. 2007. Evaluation of seasonal changes in chemical composition and antibacterial activity of Elionurus muticus (Sprengel) O. Kuntze (Gramineae). Quim. Nova. 30: 370-373.

HEYDORN, S., T. MENNÉ, K. E. ANDERSEN, M. BRUZE, C. SVEDMAN \& I. R. WHITE. 2003. Citral, a fragrance allergen and irritant. Contact dermatitis 49: 32-36.

KELCHNER, S. A. 2000. The evolution of non-coding chloroplast DNA and its application in plant systematics. Ann. Missouri Bot. Gard. 87: 482-298.

KOLB, N., D. FERRERA, E. KOLB, R. RODRIGUEZ, L VIVERO. 2007. Evaluación de la aptitud del espartillo para su explotación comercial. Misiones: Universidad Nacional de Misiones. Available at: $<$ http:// www.unam.edu.ar/index.php?option=com content\&task=view\&id=243\&Itemid $=123>$.

KOLB, E., N. KOLB, D. J. FERREYRA, R. F. ULIANA, L. S. CELAYA, C. PUGLISI, R. A. MIÑO \& J. HUK. 2012. Poaceas de Misiones: quimiotipos de Elionurus muticus. Dominguezia 28: 76.
KOSHIMA, F. A. T., L. C. MING \& M. O. M. MARQUES. 2006. Produção de biomassa, rendimento de óleo essencial e de citral em capim-limão, Cymbopogon citratus (DC.) Stapf, com cobertura morta nas estações do ano. Revista Bras. Pl. Med. 8: 112-116.

LIBRADO, P., \& J. ROZAS. 2009. DnaSP v5: A software for comprehensive analysis of DNA polymorphism data. Bioinformatics 25: 1451-1452.

LOVELESS, M. D. \& J. L. HAMRICK. 1984. Ecological determinants of genetic structure in plant populations. Ann. Rev. Ecol. Syst. 15: 65-95.

MAGRI, D., G. G. VENDRAMIN, B. COMPS, I. DUPANLOUP, T. GEBUREK, D. GÖMÖRY, M. LATALOWA, T. LITT, L. PAULE, J. M. ROURE, I. TANTAU, W. O. VAN DER KNAAP, R. J. PETIT \& J. BEAULIEU. 2006. A new scenario for the Quaternary history of European beech populations: palaeobotanical evidence and genetic consequences. New Phytol. 171: 199-221.

MASI, L., P. SIVIERO, C. ESPOSITO, D. CASTALDO, F. SIANO \& B. LARATTA. 2006. Assessment of agronomic, chemical and genetic variability in common basil (Ocimum basilisum L.). Eur. Food Res. Technol. 223: 273-281.

MC CAULEY, D. E. 1995. The use of chloroplast DNA polymorphism in studies of gene flow in plants. Trends Ecol. Evol. 10: 198-202.

MUCHUGI, A., G. M. MULUVI, R. KINDT, C. A. C. KADU, A. J. SIMONS \& R. H. JAMNADASS. 2008. Genetic structuring of important medicinal species of genus Warburgia as revealed by AFLP analysis. Tree Genet. Genomes. 4: 787-795.

MUCHUWETI, M., E. KATIVU, A. R. NDHLALA, C. MUPURE, C. CHIDEWE \& M. A. N. BENHURA. 2007. Phenolic content and phospholipids peroxidation inhibition by methanolic extracts of two medicinal plants: Elionurus muticus and Hypoxis hemerocallidea. Afr. J. Biochem. Res. 1: 137-141.

NICORA, E. G. \& Z. RÚGOLO DE AGRASAR. 1987. Los géneros de gramíneas de América Austral. Ed. Hemisfério Sur, Buenos Aires.

NODARI, R. O. \& M. P. GUERRA. 2000. Aspectos genéticos e moleculares da produção vegetal. En: SIMÔES, C. M. O. et al. Farmacognosia: da planta ao medicamento: pp. 25.38. 2.ed. Editora da UFSC, Florianópolis.

PARKER, P. G., A. A. SNOW, M. D. SCHUG, G. C. BOOTOM \& P. A. FUERST. 1998. What molecules can tell us about populations: choosing and using a molecular marker. Ecology 79: 361-382.

PEAKALL, R. \& P. E. SMOUSE. 2006. GENALEX 6: Genetic Analysis in Excel. Population genetic software for teaching and research. Mol. Ecol. Notes. 6: 288-295. 
PETIT, R. J., A. KREMER \& D. B. WAGNE. 1993. Finite island model for organelle nuclear genes in plants. Heredity 71: 630-641.

PRITCHARD, J. K., M. STEPHENS \& P. DONNELLY. 2000. Inference of population structure using multilocus genotype data. Genetics 155: 945-959.

RENVOIZE, S. A. 1978. Studies in Elionurus (Gramineae). Kew Bull. 32: 665-672.

SABINI, L. I., P. CORDERO GABRIELI, C. V. TORRES, F. M. ESCOBAR, M. CACCIABUE, M. ROVERA \& N. KOLB. 2006. Study of the citotoxic and antifungal activity of the essencial oil of Elionurus muticus against Candida spp. Mol. Med. Chem. 11: 31-3.

SCHAAL B. A., D. A. HAYWORTH, K. M. OLSEN, J. T. RAUSCHER \& W. A. SMITH. 1998. Phylogeographic studies in plants: problems and prospects. Mol. Ecol. 7: 465-474.

SCRAMIN S., M. L. SAITO, A. POTT \& M. ORTIZ MAYO MARQUES. 2000. Essential oil of Elionurus muticus (Sprengel) O. Kuntze (Gramineae). JEOR. 12: 298-300.

SHANNON, C. \& W. WEAVER. 1949. The mathematical theory of communication. University of Illinois Press, Urbana.

SHASANY, A. K., M. P. DAROKAR, S. DHAWAN, A. K. GUPTA, S. GUPTA, A. K. SHUKLA, N. K. PATRA \& S. P. S. KHANUJA. 2005. Use of RAPD and AFLP markers to identify inter and intra-specific hybrids of Mentha. J. Hered. 96: 542-549.

SHAW, J., E. B. LICKEY, J. T. BECK, S. B. FRAMER, W. LIU, J. MILlER, K. C. SIRIPUN, C. T. WINDER, E. E. SCHILLING \& R. L. SMALL. 2005. The tortoise and the hare II: relative utility of 21 noncoding chloroplast DNA sequences for phylogenetic analysis. Am. J. Bot. 92: 142-166.

SIMMONS, M.P. \& H. OCHOTERENA. 2000. Gaps as characters in sequence-based phylogenetic analyses. Syst. Biol. 49: 369-381.
SORENG, R. J., P. M.PETERSON, K. ROMASCHENKO, G. DAVIDSE, F. O.ZULOAGA, E. J. JUDZIEWICZ, T. S. FILGUEIRAS, J. I. DAVIS \& O. MORRONE. 2015. A worldwide phylogenetic classification of the Poaceae (Gramineae). J. Syst. Evol. 53: 117-137.

SORENG, R. J., P. M.PETERSON, K. ROMASCHENKO, G. DAVIDSE, J. K. TEISHER, L. G. CLARK, P. BARBERA, L. J. GILLESPIE \& F. O. ZULOAGA. 2017. A worldwide phylogenetic classification of the Poaceae (Gramineae) II: and update and a comparison of the two 2015 classifications. J. Syst. Evol. 55: 259-290.

STUCKERT, T. 1904. Primera contribución al conocimiento de las Gramináceas Argentinas. Anales Mus. Nac. Buenos Aires 11: 43-161.

TAMURA K., D. PETERSON, N. PETERSON, G. STECHER, M. NEI \& S. KUMAR. 2011. MEGA5: Molecular evolutionary genetics analysis using maximum likelihood, evolutionary distance, and maximum parsimony methods. Mol. Biol. Evol. 28: 2731-2739.

TREDGOLD, M. H. 1986. Food plants of Zimbabwe. Mambo Press, Zimbabwe.

VIDAL, A. A. 1954. Estudio químico de una gramínea argentina "Elionurus viridulus Hackel". Revista Fac. Agr. 302: 167-180.

WATSON, L. \& J. M. DALLWITZ. 1992. The grass genera of the World. CAB International, Wallingford.

YAMAMOTO, P. Y. 2006. Interação genótipo $\times$ ambiente na produção e composição de óleos essenciais de Lippia alba (Mill.) N. E. Br. Dissertação (Mestrado em Agricultura Tropical e Subtropical) - Instituto Agronômico, Campinas.

Recibido el 27 de diciembre de 2017, aceptado el 15 de mayo de 2018. Editor: Gabriel Bernardello. 